\title{
A QUASI-INVARIANCE THEOREM FOR MEASURES ON BANACH SPACES
}

\author{
BY
}

DENIS BELL

\begin{abstract}
We show that for a measure $\gamma$ on a Banach space directional differentiability implies quasi-translation invariance. This result is shown to imply the Cameron-Martin theorem. A second application is given in which $\gamma$ is the image of a Gaussian measure under a suitably regular map.
\end{abstract}

1. Introduction. A (Borel) measure on a vector space is said to be quasiinvariant under a vector $h$ if the class of its null sets is preserved by $h$-translation. It is known that for a measure on $\mathbf{R}^{n}$ quasi-invariance under all translations implies equivalence to the Lebesgue measure (see $\S 4.3$ ). This result has no analogue in infinite dimensions. However knowledge of the quasi-invariance properties of a measure on an infinite dimensional vector space provides important information about the structure of the measure.

In the work described here we show that for a measure on a Banach space differentiability in a direction $h$ implies quasi-invariance under $h$. Two applications of this theorem are given. In the first $\gamma$ is assumed to be a Gaussian measure and the Cameron-Martin theorem is obtained. In the second application we consider the projection of a Gaussian measure under a map satisfying certain regularity conditions.

This work was motivated by the recent development of the Malliavin Calculus. Malliavin's theory provides a method of establishing the directional differentiability of the measure induced on $\mathbf{R}^{n}$ by the solution of a stochastic differential equation (see [4], for example). The corresponding result obtained by an extension of this program to an infinite dimensional setting would produce measures on a Banach space $E$ which are differentiable in a dense linear subspace. In conjunction with the present theorem, this would then establish the quasi-invariance of the induced measures on $E$.

There are many different notions of measure differentiability. The definition adopted here is closely related to Skorohod's Logarithmic Differentiability (see [5, p. 121]) and was motivated by Malliavin's result.

Suppose then that $\gamma$ is a finite Borel measure on a Banach space $E$, and $h$ is an element of $E$. We will say that a function $\phi$ defined on $E$ is $h-C^{1}$ if $\phi$ has a Gâteaux derivative in the direction $h$, denoted by $D_{h} \phi$, and for almost all $x$ the function $\phi(x+\cdot)$ is continuous along the line spanned by $h$. The measure $\gamma(h+\cdot)$ will be denoted by $\gamma_{h}$.

Received by the editors October 15, 1983 and, in revised form, June 8, 1984 and November 10, 1984.

1980 Mathematics Subject Classification. Primary 46G12. 
DEFinition. $\gamma$ is $h$-differentiable if there exists an $L^{2}$ function $X$ such that the relation

$$
\int_{E} D_{h} \phi(x) d \gamma(x)=\int_{E} \phi(x) X(x) d \gamma(x)
$$

holds for all $h-C^{1}$ functions $\phi$ with $\phi$ and $D_{h} \phi$ bounded.

2. In this section we prove the following

THEOREM. Suppose that $\gamma$ is h-differentiable, $X(x+\cdot)$ is continuous in the direction $h$ for almost all $x$ and that

$$
\sup _{t \in[0,1]}|X(x+t h)|^{4} \quad \text { and } \sup _{s, t \in[0,1]} \exp -4 \int_{s}^{t} X(x+u h) d u
$$

are locally integrable. Then $\gamma$ is quasi-invariant under $h$ with

$$
\frac{d \gamma_{h}}{d \gamma}=\exp -\int_{0}^{1} X(x+u h) d u \quad \text { a.s. }
$$

The proof of the theorem requires the following

LEMMA. If $\gamma$ is $h$-differentiable, then (1) holds for every $h-C^{1}$ function $\phi$ such that $\phi$ and $\sup _{t \in[0, \varepsilon]} \mid D_{h} \phi(x+$ th $) \mid$ are in $L^{2}$ for some $\varepsilon>0$.

PROOF. We first assume that $\phi$ is bounded. For each $N$ let

$$
\phi_{N}(x)=N \int_{0}^{1 / N} \phi(x+u h) d u .
$$

Then $\phi_{N}$ is bounded, $h-C^{1}$ and $D_{h} \phi_{N}$ is also bounded. Hence (1) holds for $\phi_{N}$. Now

$$
\int_{E} D_{h} \phi_{N}(x) d \gamma(x)=\int_{E} N\left[\phi\left(x+\frac{h}{N}\right)-\phi(x)\right] d \gamma(x)
$$

As $N \rightarrow \infty$ this converges to

$$
\left.\frac{d}{d t} \int_{E} \phi(x+t h) d \gamma(x)\right|_{t=0}
$$

provided this exists. For $t \in[0, \varepsilon]$

$$
\begin{aligned}
\int_{E}[\phi(x+t h)-\phi(x)] d \gamma(x) & =\int_{E} \int_{0}^{t} D_{h} \phi(x+u h) d u d \gamma(x) \\
& =\int_{0}^{t} \int_{E} D_{h} \phi(x+u h) d \gamma(x) d u .
\end{aligned}
$$

Here we have used Fubini's theorem to interchange orders of integration. Differentiating with respect to $t$ at $t=0$ in (3) gives $(2)=\int_{E} D_{h} \phi(x) d \gamma(x)$. Hence we have shown that as $N \rightarrow \infty$

$$
\int_{E} D_{h} \phi_{N}(x) d \gamma(x) \rightarrow \int_{E} D_{h} \phi(x) d \gamma(x) .
$$

Now $\phi_{N} \rightarrow \phi$ in $L^{2}$ so taking the limit over $N$ in (1), applied to each $\phi_{N}$ shows that (1) also holds for $\phi$. 
We will now remove the boundedness assumption on $\phi$. Suppose $\phi$ satisfies the assumption of the lemma. Let $B_{M}: \mathbf{R} \rightarrow[0,1]$ be a sequence of smooth functions such that

(i) $B_{M}(x)=1$ if $|x| \leq M$.

(ii) $B_{M}(x)=0$ if $|x| \geq M+1$.

(iii) $D B_{M}(x)$ is uniformly bounded in $M$ and $x$.

Then for each $M, \psi_{M}=\phi B_{M} \circ \phi$ is a bounded function which also satisfies the hypothesis of the lemma. So (1) holds for $\psi_{M}$. Now taking the limit over $M$ and using the fact that $\phi, D_{h} \phi$ and $X$ are in $L^{2}$ gives (1) for $\phi$.

PROOF OF THE THEOREM. It suffices to show that, for every bounded real valued continuous function $\theta$ with bounded support,

$$
\int_{E} \theta(x) d \gamma(x)=\int_{E} \theta(x+h) \exp -\int_{0}^{1} X(x+u h) d u d \gamma(x) .
$$

We will show this under the additional assumptions that $\theta$ is $h-C^{1}$ and has $D_{h} \theta$ bounded. (4) will then follow in the required generality by approximating $\theta$ by a sequence of functions $\theta_{N}$ defined as in the proof of the lemma.

For ease of notation, let us denote $\exp -\int_{0}^{t} X(x+u h) d u$ by $E(t)$. We now define a function $g$ from $[0,1]$ into $\mathbf{R}$ by

$$
g(t)=\int_{E} \theta(x+t h) E(t) d \gamma(x)
$$

It is clear that $g$ is continuous. We will prove that $g$ is constant by showing that $g^{\prime} \equiv 0$ on $(0,1)$. First note that the integrand in (5) is differentiable in $t$ with derivative

$$
G(x, t)=\left[D_{h} \theta(x+t h)-\theta(x+t h) X(x+t h)\right] E(t) .
$$

As $G(x, t)$ is continuous in $t$ and $\sup _{t \in[0,1]}|G(x, t)| \in L^{1}$ it follows by the Dominated Convergence Theorem that $\int_{E} G(x, t) d \gamma(x)$ is also continuous in $t$. Hence we may differentiate under the integral sign in (5) to obtain $g^{\prime}(t)=\int_{E} G(x, t) d \gamma(x)$.

Now observe that for almost every $x, E(t)$ is $h$-differentiable and

$$
D_{h} E(t)=E(t)[X(x)-X(x+t h)] .
$$

Substituting this into (6) gives

$$
G(x, t)=D_{h} \theta(x+t h) E(t)+\theta(x+t h) D_{h} E(t)-\theta(x+t h) E(t) X(x) .
$$

Now the function $\phi(x)=\theta(x+t h) E(t)$ satisfies the conditions on $\phi$ in the lemma, so (1) holds. Since the $h$-derivative of $\phi$ consists of the first two terms on the righthand side in (7), integrating with respect to $\gamma$ in (7) gives $\int_{E} G(x, t) d \gamma(x)=0$ as required.

3. We now give two applications of this result.

(1) Suppose that $(i, H, E)$ is an abstract Wiener space and $\gamma$ is Gaussian measure on $E$. Let $E^{*}$ be embedded in $E$ in the usual way, via the inclusions

$$
E^{*} \stackrel{i^{*}}{\hookrightarrow} H^{*} \sim H \stackrel{i}{\hookrightarrow} E
$$

(See [2] for details of abstract Wiener spaces.) It is shown in [3] that if $h \in H$, then $\gamma$ is $h$-differentiable and $X(x)=\langle h, x\rangle$, where $\langle h, \cdot\rangle$ is an extension of the 
inner product on $H$ to an $L^{2}$ random variable on $E$. In this case, for almost all $x$, $X(x+t h)=\langle h, x\rangle+t|h|_{H}^{2}$. This implies the continuity required by the theorem together with the inequalities

$$
\begin{gathered}
\sup _{t \in[0,1]}|X(x+t h)|^{4} \leq\left[|\langle h, x\rangle|+|h|_{H}^{2}\right]^{4}, \\
\sup _{s, t \in[0,1]} \exp \left\{-4 \int_{s}^{t} X(x+u h) d u\right\} \leq \exp \left\{4|\langle h, x\rangle|+2|h|_{H}^{2}\right\} .
\end{gathered}
$$

Since $\langle h, x\rangle$ has a normal distribution, both (8) and (9) are integrable. So the theorem implies that $\gamma$ is quasi-invariant under $h$ with

$$
\frac{d \gamma_{h}}{d \gamma}=\exp -\left\{\langle h, x\rangle+1 / 2|h|_{H}^{2}\right\} \quad \text { a.s. }
$$

This is the Cameron-Martin theorem.

(2) Assuming the same notation as in example (1), suppose that $T$ is an injective map from $E$ to a second Banach space $K$. Suppose that for some $h \in K$ the following conditions are satisfied:

(i) For any $y \in \operatorname{Range} T^{\text {: }}$ and $t \in \mathbf{R}, y+t h \in \operatorname{Range} T$ and $T^{-1}(y+t h)$ is continuous in $t$.

(ii) $T$ is $C^{1}$ and there exists a $C^{1}$ map $S$ from $E$ into $E^{*}$ such that $D T(x) S(x) \equiv$ $h$.

(iii) The maps $T, T^{-1}, S$ and $x \rightarrow$ Trace $_{H} D S(x)$ are bounded on bounded sets. ${ }^{1}$

Let us denote the induced measure on $K, T(\gamma)$ by $\nu$. The following calculation shows that $\nu$ is $h$-differentiable with directional derivative

$$
X(x)=\left\langle S\left(T^{-1} x\right), T^{-1} x\right\rangle-\operatorname{Trace}_{H} D S\left(T^{-1} x\right), \quad \nu \text {-a.s. }
$$

where $\langle$,$\rangle denotes the pairing between E^{*}$ and $E$. Since under conditions (i), (ii) and (iii) $X$ satisfies the required hypotheses the theorem implies that $\nu$ is quasiinvariant under $h$.

To establish (10) we proceed as follows:

$$
\begin{aligned}
\int_{K} D_{h} \phi(x) d \nu(x) & =\int_{E} D_{h} \phi(T x) d \gamma(x)=\int_{E} D(\phi \circ T)(x) S(x) d \gamma(x) \\
& =\int_{E} \phi \circ T(x)\left\{\langle S(x), x\rangle-\operatorname{Trace}_{H} D S(x)\right\} d \gamma(x) .
\end{aligned}
$$

The last equality is obtained by applying the divergence theorem (see $[\mathbf{1}]$ ). Hence (10) follows.

4. Concluding remarks. 1. The conclusion of the theorem may be stated in the form: $\gamma$ is quasi-invariant under $t h$, for any $t \in[0,1]$, with

$$
\frac{d \gamma_{t h}}{d \gamma}=\exp -\int_{0}^{t} X(x+u h) d u \quad \text { a.s. }
$$

2. Skorohod has studied the relationship between directional differentiability and quasi-translation for a measure on a Hilbert space [5, $\S \S 21-23]$. In this setting he

\footnotetext{
${ }^{1}$ By way of explanation here we remark that as $S$ is a $C^{1}$ map from $E$ to $E^{*}$, then, for each $x, D S(x)$ restricts to give a trace class operator on $H$.
} 
proves a theorem similar to ours under weaker hypotheses. However the techniques used in Skorohod's work depend upon the Hilbert structure of the measure space and hence do not apply in the present situation unless $E$ is Hilbertizable.

3. If $E$ is finite dimensional, then $\gamma$ can be shown to be $h$-differentiable if (1) holds for all $C^{1}$ functions with compact support. Suppose that, for some orthonormal basis $e_{1}, \ldots, e_{n}, \gamma$ has continuous directional derivatives $X_{1}, \ldots, X_{n}$. Then the theorem implies that $\gamma$ is quasi-invariant under all translations. An elementary argument then gives the result that $\gamma$ is equivalent to the Lebesgue measure with density function

$$
F(x)=C \exp \left[-\sum_{i=1}^{n} x_{i} \int_{0}^{1} X_{i}(u x) d u\right] \quad \text { a.s. }
$$

where $C$ is a constant.

\section{REFERENCES}

1. K. D. Elworthy, Gaussian measures on Banach spaces and manifolds, Global Analysis and Applications, Vol. 2, International Atomic Energy Agency, Vienna, 1974, pp. 151-166.

2. L. Gross, Abstract Wiener space, Proc. Fifth Berkeley Sympos. Math. Statist. and Probability, part 1, University of California, 1965, pp. 31-42.

3.

4. P. Malliavin, Stochastic calculus of variations and hypoelliptic operators, Proc. Internat. Conf. on Stochastic Differential Equations of Kyoto, 1976, pp. 195-263.

5. A. V. Skorohod, Integration in Hilbert space, Springer-Verlag, New York, 1974.

Department of Mathematics, The University of Texas, Austin, Texas 78712

Current address: Department of Mathematics, Suffolk University, Boston, Massachusetts 02114 